\title{
A tumor microenvironment destroyer for efficient cancer suppression
}

Hai Yao ${ }^{1}$, Kaikai Xu ${ }^{1}$, Jiahong Zhou, Lin Zhou *, Shaohua Wei *

College of Chemistry and Materials Science, Jiangsu Key Laboratory of Biofunctional Materials, Jiangsu Collaborative Innovation Centre of Biomedical Functional Materials, Key Laboratory of Applied Photochemistry, Nanjing Normal University, Wenyuan Road, Nanjing (210023), China.

${ }^{1}$ Equal the first author.

* Corresponding author. E-mail: zhoulin@njnu.edu.cn (L. Zhou); shwei@njnu.edu.cn (S. H. Wei); Tel: +86-025-8589-1761.

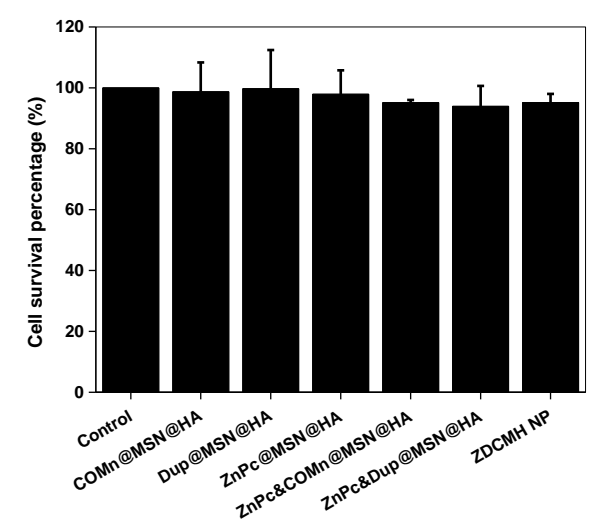

Figure S1. The dark toxicity of control, COMn@MSN@HA, Dup@MSN@HA, ZnPc@MSN@HA, ZnPc\&COMn@MSN@HA, ZnPc\&Dup@MSN@HA and ZDCMH NP.

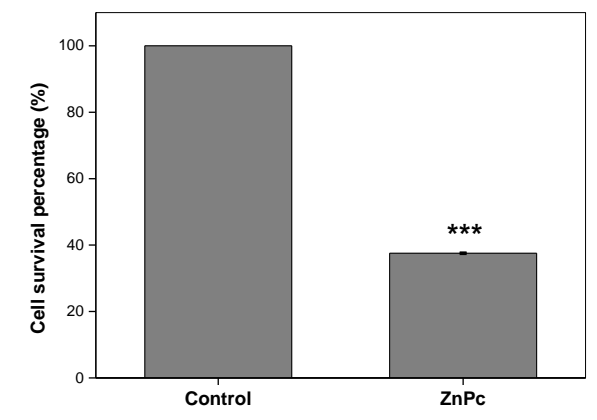

Figure S2. The photodynamic therapy effect of ZnPc to fibroblasr HFL-1 cells. 

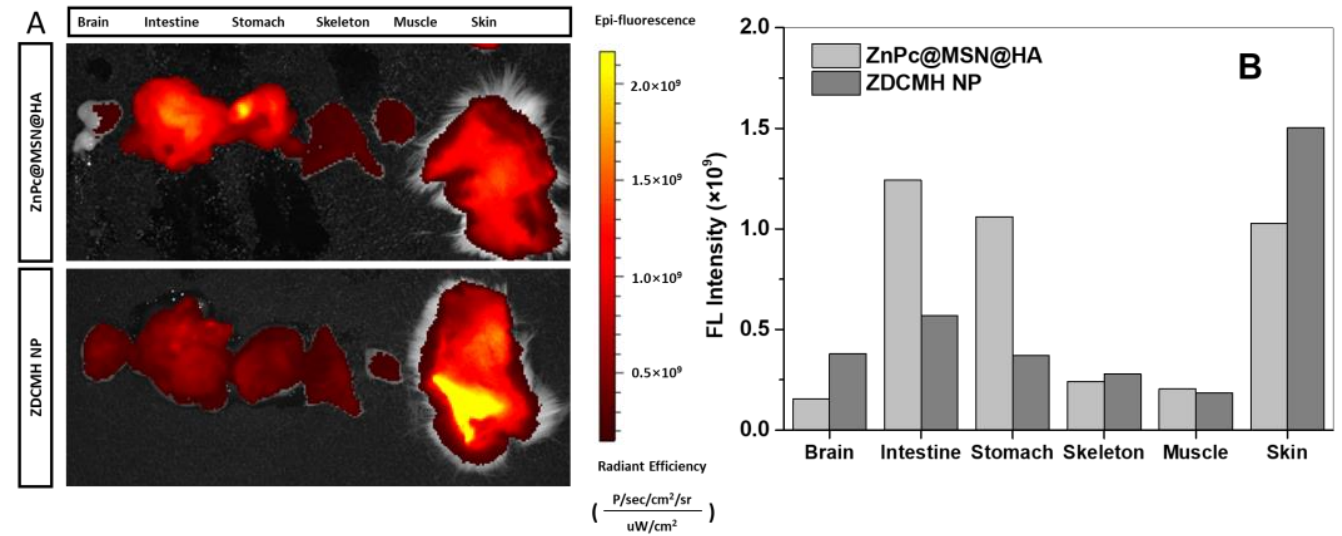

Figure S3. (A) The fluorescence signal of tissues from tumor bearing mice treated by ZnPc@MSN@HA and ZDCMH NP; (B) the relative fluorescence intensity of tissues from tumor bearing mice treated by ZnPc@MSN@HA and ZDCMH NP. 